\title{
Acoso Laboral vs Síndrome de Cronos. Una carga emocional ética si no se sabe controlar
}

\author{
Diamela Nava \\ Universidad Rafael Belloso Chacín, Maracaibo, Venezuela \\ Correo electrónico autor: diamelanava@gmail.com \\ Recepción: 25 Septiembre 2017 \\ Aprobación: 25 Noviembre 2017
}

\begin{abstract}
Resumen
El objetivo de la presente investigación tuvo como finalidad analizar el acoso laboral vs el síndrome de cronos como dos factores que afectan emocionalmente la salud mental del individuo. El estudio se basa en una metodología de tipo interpretativa, utilizando un diseño no experimental, mediante un análisis documental. Finalmente se logró considerar que el objetivo fundamental de ambos riesgos psicosociales es transformar moralmente al trabajador y destruirlo lentamente a través del abuso de poder, acoso moral, entre otros. No obstante, el jefe-cronos puede estar influenciado por el temperamento, carácter con sentimientos de inseguridad, miedo a ser desplazado, para poder sentirse identificado como una persona admirada en la organización para hacer valer su autoridad.
\end{abstract}

Palabras clave: Acoso, Síndrome de Cronos, Dignidad Humana. 


\title{
Labor Acoso vs Chronos Syndrome. An emotional ethical charge if you cannot control
}

\begin{abstract}
The objective of the present investigation was to analyze the work harassment vs the syndrome of cronos as two factors that emotionally affect the mental health of the individual. The study is based on an interpretative methodology, using a non-experimental design, through a documentary analysis. Finally, it was possible to consider that the fundamental objective of both psychosocial risks is to morally transform the worker and to destroy it slowly through abuse of power, moral harassment, among others. However, the boss-chronos may be influenced by temperament, character with feelings of insecurity, fear of being displaced, to be able to feel identified as a person admired in the organization to assert its authority.
\end{abstract}

Keywords: Harassment, Cronos Syndrome, Human Dignity.

\section{Introducción}

A través del tiempo en las organizaciones se han descubierto en múltiples formas de liderar desde aquel que sustentaba su autoridad de manera abusiva hasta el origen divino de aquellos que lo ejecutaron por las fuerzas de las armas. De esta manera, la evolución de las organizaciones algunas veces resultan ser improductivas, anárquica e incluso caótica cuando carece de una figura capaz de inculcar una cultura organizacional a través de unos valores éticos ejemplares, para proporcionar congruencia en las relaciones y actividades.

De esta manera, cada individuo constituye un mundo, las experiencias son propias e incompatibles en virtud que, un grupo de personas están compuestas por unas características particulares, cualidades o potencialidades, defectos, entre otros. Por tanto, el manejo de personas es quizás la actividad más difícil que pueda realizar el ser humano.

Sin embargo, según Grettchen (2011), la gestión organizacional coordinada por un jefe autocrático puede padecer del síndrome de cronos que lo conlleva a sentir un miedo patológico para que otros no lo puedan superar profesionalmente y tampoco siente humildad para que los demás aprendan lo que sabe hacer, todo ello con el propósito de tener una necesidad excesiva de mantenerse seguro, no obstante; de sentir una pasión enfermiza por su puesto de trabajo y así no ser desplazado o sustituido, creando en la organización puestos inamovibles.

En este sentido, el síndrome de cronos está relacionado con otros comportamientos patológicos como el acoso laboral, egocentrismo, 
autoengaño, adicción al trabajo y el presentismo. Asimismo, detrás de este comportamiento se puede esconder un agresor con conductas degradantes hacia los demás, con el objetivo de sacarlo de la empresa, especialmente ataca aquellos trabajadores con mayor nivel de preparación y eficiencia.

Atendiendo a estas consideraciones, el perfil de un jefe-cronos ocupa un puesto con relativa importancia; desde un punto de vista emocional posee una baja autoestima, desconfianza hacia el equipo de trabajo, con poca preparación académica, carece de potencialidades y pueden tener cierto grado de antigüedad en la empresa, pero trata de rodearse de gente sumisa para manipularla y manejarla a su manera.

\section{Acoso laboral: un riesgo psicosocial que afectan la salud mental de la victima}

Sobre la base de las ideas expuestas, las víctimas de acoso van más allá al afirmar que el acto violento constituye un atentado contra la integridad moral y ética de la persona, en virtud que la actuación del acosador va dirigida de modo directo para someter y manipular al mismo, con la intención de echarlo del territorio aunque algunas veces el psicópata no consiga sacarlo de la empresa, por lo menos le queda la satisfacción de hacerle la vida imposible con la mala impresión de hacer pasar al acosado como un trabajador incompetente, improductivo $\mathrm{y}$ problemático.

No obstante, es por ello que, una táctica muy utilizada es la provocación continuada, a través de la cual se intenta que la víctima o acosado, debido a la tensión o estrés al que vive sometido a diario, acabe "explotando" y traicionándose a sí mismo, bien de palabra o acto, con lo que ya se ha logrado el pretexto para la expulsión y así el acosador consigue el objetivo perseguido. A este respecto, según Verona \& Santana (2012), el individuo durante el proceso de acoso en el ámbito laboral algunas veces son variados y suelen utilizarse una combinación de gestos, palabras, o cualquier tipo de actitud negativa para amedrentar a la víctima, a saber:

- Gritar, insultar y humillar a la víctima cuando la misma está sola o acompañado de otras personas, con el fin de ridiculizarlo.

- Asignarles proyectos con plazos de tiempo inalcanzables de cumplir.

- Quitarle actividades que son importantes en su área de trabajo y colocarle otras tareas rutinarias sin interés, ello con la 
finalidad de desarrollar en la víctima el Síndrome del Quemado o incluso aplica la técnica del ninguneo, es decir; "Matar a la víctima con la indiferencia y el menosprecio de no tomarlo en cuenta en ninguna actividad, con el objetivo que el mismo se aburra y renuncie a la empresa.

- Manipular y retener la información para inducirle a un error durante su desempeño laboral y acusarle ante su superior por falta de negligencia y deficiencia en sus actividades laborales.

- Difamar o levantar falsos en contra de la víctima, extendiendo comentarios destructivos por toda la empresa que influyen de manera negativa en contra de su reputación o imagen profesional.

- Ignorar por motivos de envidia sus éxitos profesionales ante otras personas.

- La víctima es criticada continuamente por sus trabajos, ideas, propuestas, entre otros factores, convirtiéndose en objeto de burlas.

- El acosador puede castigar duramente por cualquier iniciativa en el marco de sus responsabilidades y atribuciones, lo que conlleva a una supuesta falta de obediencia ante la jerarquía.

- Otra técnica utilizada es invadir y otra veces divulgar la vida privada de la víctima, accediendo a su correo electrónico, teléfono privado, documentos referentes a su vida personal, entre otros.

- En algunos casos se han visto situaciones de líderes acosadores que despiden injustificadamente al trabajador, sea por discriminación sexual, religiosa y/o política.

- Ausencia de sentimientos y culpabilidad. Atendiendo a estas consideraciones, la víctima de acoso laboral no siempre le resulta fácil probar o demostrar los abusos desarrollados en su contra sin justificación alguna, a pesar de la profunda huella psicológica que esté dejando en su salud mental, que se estén presentando por este tipo de violencia que en algunos casos el acosador siempre trata de no dejar ninguna evidencia y así evitar que la persona atacada pueda defenderse y dejarla como mentirosa por no tener las pruebas suficientes, siendo la palabra de la víctima contra el acosador o psicópata.

Por tanto, según Martínez, Muñiz, 
Camino, Torres y Burón, (2012), asumen que para la víctima por muy difícil que sea demostrar que ha sido lastimado emocionalmente por el acoso laboral es su deber como ser humano, dar a conocer el daño hasta las últimas instancias por el bienestar de su salud mental y física, aunque su reputación y autoestima esté seriamente afectada.

De esta manera, en el ambiente laboral cuando se presentan situaciones inestables con el personal y que puedan perjudicar moral y psicológicamente al personal es necesario que la organización busque especialistas en el área de la psicología para mantener la armonía y alta autoestima de los trabajadores.

No obstante, según Martínez (2012) el acoso también afecta directamente al acosador en su bienestar mental y vida personal, que conlleva a fortalecer sus malestares físicos. Sin embargo, cualquier persona puede ser víctima de este tipo de violencia emocional, por lo que es importante analizar los factores que estén presentes para llevar a cabo el acoso.

En este sentido, el acoso laboral como avalancha de intimidaciones que conlleva a una incidencia en contra de la dignidad del ser humano, buscando el desgaste psicológico de aquellos trabajadores molestos durante un periodo de tiempo prolongado mediante conductas sociópatas, intimidatorias, y hostigadoras, de parte de un agresor o grupo de agresores que se presenta a través de un conjunto de conductas premeditadas, y extremadamente sutil con un objetivo claro que es destruir a la víctima.

De esta manera, el mayor problema en las organizaciones es que la víctima de violencia psicológica algunas veces por causas del miedo, pasa a sufrir la situación en silencio hasta que llega el momento en que presenta la pérdida de control del bienestar físico y mental. No obstante, es un reflejo de las deficiencias de las organizaciones debido a la falta de información o desconocimiento y por no inculcar un sistema de valores necesarios para el bienestar común de todos los que la integran.

Síndrome de Cronos: una acción deliberada por la necesidad de obtener poder

El ser humano es complejo e imprescindible en su comportamiento, de esta manera, a lo largo del tiempo se ha demostrado de lo que es capaz de hacer para satisfacer su necesidad de ambición de poder. No obstante, según Bedoya \& Velásquez (2014) señalan que el líder con síndrome de cronos cuando presenta 
una necesidad de poder, solo se esmeran en analizar sus emociones en los logros personales pero no ocupan el mayor tiempo posible para dirigir y motivar a su equipo, realizando todas las actividades laborales a la vez sin delegar, por temor a que cualquier miembro su equipo desarrolle mejor las funciones. No obstante, este tipo de comportamiento conlleva a desmotivar emocionalmente al resto del equipo con el propósito de sentirlos ignorados o que no son capaces para desarrollar de manera eficiente las actividades laborales.

Asimismo, el líder con cronos se satisface a través de ciertos riesgos psicosociales como el síndrome del burnout, acoso laboral, para controlar a sus subalternos, con la finalidad de evitar el crecimiento personal en la organización.

Sin embargo, una de las características principales que debe poseer un gerente o líder en la organización es la pasión por el trabajo, es decir cuando la persona siente amor por lo que hace; por tal motivo esta actitud tiene sus pro y sus contras; el líder se vuelve más productivo, valorado, consigue grandes logros a base de retos, llevándolo al éxito, por otro; en algunos casos la pasión por el trabajo a través del tiempo se puede convertir en obsesión o amor enfermizo sintiéndose indispensable en cuanto al desarrollo de sus actividades al pensar que es el único que puede realizarlas, con la finalidad de sentir adicción por el trabajo y estrés.

De esta manera, en las organizaciones el poder se obtiene en la medida que se asciende de cargo, a través del esfuerzo, dedicación, y una vez alcanzado es difícil aceptar que puede perderse al generar miedo al momento de delegar funciones, el cual conlleva al individuo a realizar cualquier cosa para evitar ser desplazado o sustituido.

En este sentido, según Sue y Sue (2010), cronos significa, miedo a ser desplazados del poder, pero en la mitología griega Cronos tenía tanta sed de poder que atentó contra su padre Urano, con la intención de ocupar su lugar a través de manipulaciones y engaños, debido a que éste era el amo y señor del universo. Sin embargo, Urano mal herido, le maldijo al experimentar la misma suerte en manos de sus hijos, por lo que Cronos decidió devorarlos al nacer, con el objetivo de alcanzar su propósito sin importar el daño que podría ocasionar, no obstante, y gracias a la astucia de la diosa Era, uno de sus hijos, Zeus, logró salvarse de tan cruel destino dando cumplimiento años después a la maldición.

En virtud de estas razones, en el ámbito 
gerencial, el síndrome decronos esuna enfermedad mortal porque no distingue tamaño, género, o nacionalidad por tal motivo, resulta en la mayoría de los casos se lleva a cabo de manera progresiva. Sin embargo, el miedo y la inseguridad en el líder con cronos, se complementan en los altos puestos gerenciales presentando una necesidad de poder aferrándose a ellos, por motivos de inseguridad a ser desplazado por algún miembro.

Asimismo, según Cestaus (2002) no obstante, en las organizaciones siempre estará presente la preocupación y el temor a ser desplazado por cualquier miembro, en aquellos donde la actuación del líder cronos se incline a impedir el crecimiento de sus trabajadores podría decirse que se está en presencia del ahora llamado Síndrome de Cronos. En virtud de estas razones, las organizaciones no tienen problemas con el tipo de gerencia sino en la cultura empresarial que solamente se basa en el rendimiento de los trabajadores y dejando de lado el clima organizacional.

El Síndrome de Cronos no es más que la acción deliberada del líder para estancar a su personal por temor a ser desplazado o sustituido, evitando el crecimiento de los mismos al utilizar técnicas de riesgo psicosocial como la manipulación, el psicoterror, entre otros; generando preocupación, ansiedad, frustración, estrés, desconfianza al creer que la organización desea despedirlo o bajarlo a un puesto inferior y algunas veces posee actitudes perversas utilizando la manipulación, acoso laboral, con el propósito de eliminar a la persona o personas que tengan cierto potencial para ocupar cargos de mayor responsabilidad convirtiéndose en un enemigo letal de quienes lo rodean.

En este marco de ideas, según Bedoya \& Velásquez (2014) esta “enfermedad gerencial o riesgo psicosocial" tiene unos síntomas particulares que pueden ser listados de la manera siguiente:

- Está presente en cualquier organización, independientemente de su tamaño y función.

- Se lleva a cabo a través de personas con altos cargos y de mayor responsabilidad.

- No importa la edad, el sexo de la persona, usualmente esto se lleva cabo a través de personas con mucha preparación y experiencia laboral pero a nivel emocional son personas con inseguridad y con un grado de egocentrismo muy elevado. 
- El síndrome de cronos por parte del gerente inhibe en los demás el miedo al crecimiento personal, anulando las potencialidades en cuanto a conocimientos que posea el equipo de trabajo para ejercer puestos de alta jerarquía, también puede afectar el clima y la cultura organizacional de la empresa.

En este sentido, el Síndrome de Cronos se evidencia con mayor facilidad en aquellas organizaciones donde las posibilidades de desarrollo en posiciones $\mathrm{o}$ en altos niveles jerárquicos, se evidencia altos estándares de talentos en el desempeño laboral entre los trabajadores por lo que el líder tiende acelerar su territorio para evitar mostrar su deficiencia, carencias, y temores que conllevan a desarrollar ciertas conductas que contaminan las relaciones interpersonales en la organización.

Por tanto, no siempre demuestran un comportamiento ético en sus acciones y decisiones al imponer en el perfilamiento de algunos miembros que puedan atentar o ser una amenaza en contra de la estabilidad de su cargo.

Sin embargo, El síndrome de cronos cuando atenta en contra de las premisas básicas del talento humano en la organización se constituye como una característica principal para generar desinterés en el trabajo afectando la productividad de todos, como lo expresa Bustillo (2011) cuando explica que los trabajadores necesitan perfeccionar su talento es decir; a través de formación, motivación, honor al mérito, entre otros.

En virtud de estas razones, la empresa debe tener el compromiso de fomentar un clima de crecimiento y desarrollo para todo el talento humano que la integra aun cuando las oportunidades de avanzar no sean muy altas o disminuyan; motivo por el cual, es importante que la entidad brinde apoyo a todo el personal, con el fin de conocer nuevos caminos que les permita seguir dentro de la misma, para seguir desarrollando las actividades con profesionalismo de manera exitosa.

Con base a lo expuesto se infiere que, el equipo de trabajo cuando observe la conducta deliberada y sin razón de parte del líder por mantenerlo siempre en la misma condición y no expresar su descontento por miedo a ser despedido puede reaccionar, expresando su descontento a expensas de que se sienta intimidado, se limita a comunicar lo estrictamente necesario, en espera de alguna oportunidad en donde pueda desligarse 
del supervisor.

Atendiendoa estasconsideraciones, sibien es cierto según Contreras (2010) el estancamiento a nivel psicológico produce una baja en la productividad del trabajador, asimismo no es menos cierto que habrá de generar sentimientos de impotencia y resentimiento, los cuales tienden a servir de barreras comunicacionales.

No obstante, ante la presencia del Síndrome de Cronos en la organización, el subordinado comienza a manifestar una conducta más orientada al miedo e inseguridad al momento de delegar funciones al percatarse de la imposibilidad de crecimiento o el nivel de estancamiento que esta conducta gerencial propicia hacia los demás, desarrollando características patológicas como, egocentrismo, autoengaño y desarrollo limitado entre otros trastornos. Según la teoría de Hirtz (2010), es posible también que presente síntomas de hostilidad, o indiferencia.

De acuerdo a ello, el Síndrome de Cronos es una conducta que atenta contra las cinco premisas básicas de la gerencia del talento humano, orientadas a ofrecer bienestar y desarrollo de toda organización, éstas son: productividad, comunicación, identificación, conducta y motivación. Sin embargo, estas premisas pueden ser comparadas con cada una de las extremidades del cuerpo humano, si alguna enfermedad la afecta, difícilmente éste podrá moverse con rapidez y acierto. En este sentido, esta conducta, que limita el crecimiento del individuo, ya sea a nivel de conocimiento; es sencillamente una afección gerencial, la cual se debe identificar, combatir y erradicar en cualquiera de las áreas laborales en la que se desenvuelve.

\section{Código de conducta ética en la gestión} organizacional: una herramienta para la armonización en las relaciones

\section{interpersonales}

En la actualidad la necesidad de adecuar las normas éticas en las organizaciones para lograr responder a las demandas y desafíos que genera un mundo cada vez más globalizado tanto en el sector público y privado, que conlleva al Talento Humano a estar sometido a continuas y complejas relaciones interpersonales. No obstante, este proceso no solo se lleva a cabo internamente en la empresa sino también externamente con la sociedad en general.

Es así como según Verona \& Santana (2014), la ética no es algo extrínseco que 
constriñe, es decir, que impone en la actividad empresarial, tampoco pertenece al ámbito privado del directivo o líder, la ética es una condición necesaria para el correcto funcionamiento de la organización que conlleva a la excelencia individual y organizacional. De esta manera, el código de conducta ética no debe ser visto como un listado de prohibiciones o reglas que traten de inhibir la superación personal, sino que permita incentivar prácticas constantes de actitudes positivas que conlleve al bienestar general en las relaciones interpersonales.

Así mismo, según Seijo y Añez (2011), la ética es una dimensión de la realidad humana que no puede quedar al margen de ninguna actividad empresarial. En virtud de estas razones, la empresa puede y debe tener conciencia, por tal motivo, los comportamientos irresponsables impiden la rentabilidad y viabilidad de la empresa a largo plazo y además debilitan la eficiencia, productividad y confianza del sistema económico o maximización de valor en la organización.

Con base a lo expuesto se infiere que, la reputación es uno de los principales activos que poseen y deben mantener las organizaciones para seguir llevando a cabo las buenas prácticas profesionales. Según la doctrina citada por
Brealey, Myers \& Marcus, (2014) plantean la importancia de tener presente el mecanismo de la buena reputación, por tal motivo, este ha sido tradicionalmente uno de los medios más indispensables para asegurar el cumplimiento en cuanto a los estándares éticos establecidos en el código de ética de la empresa.

Así, la reputación se podría definir como un ejemplo a seguir a través de ciertos comportamientos éticos y morales que se han formado desde el pasado, por ello; la misma constituye la base fundamental para predecir su comportamiento en el futuro a través de unos valores y principios éticos ejemplares.

En virtud de estas razones, las gerencias en las organizaciones actúan como encubridores de los acosadores laborales, se preocupan en la promoción de reflexiones colectivas entre sus miembros, con el fin de aportar a la consecución del bien más preciado para el ser humano como es la felicidad. En la medida en que nosotros, hijos, familiares, vecinos, amigos, comunidad y todos juntos practiquen comportamientos éticos y morales, en esa medida se logra construir la vida y el mundo que todos deseamos.

Para tal efecto, la falta de un Código de Conducta Ética para el mejoramiento en 
las relaciones en las empresas, probablemente afloren los celos profesionales, la envidia y en el peor de los casos, perjudicar a quienes producto de su esfuerzo crecen profesionalmente y son promotores del fortalecimiento de la identidad organizacional.

Con base a lo expuesto, todo lo anterior es el resultado típico del hostigamiento psicológico en el trabajo. Como complemento, según Martínez (2012) define al "acosado laboral" como una persona con elevado sentido de la ética y la justicia, muy capacitado para su trabajo, fuerte sentimiento de compañerismo, iniciativa y trabajo en equipo, además aprecio entre los compañeros de trabajo. Por tanto, este perfil es el que promueve el Código de Ética a lo interno de las organizaciones.

En síntesis, las organizaciones deben tener una serie de valores éticos y conseguir que sus empleados, de cualquier nivel, alto, o intermedio, compartan esos valores y realicen su trabajo teniéndolos presente, y en el proceso de toma de decisiones consideren la ética como un criterio más, para el bienestar en las relaciones internas y externas. Por su parte, la eficacia de los códigos éticos depende en gran parte del proceso de la ejecución del mismo y de integrar la cultura ética en el día a día.

Asimismo, según Lozano (2011), la elaboración de estos códigos deben ser un proceso participativo y dialogado en el que todos y cada uno de los miembros de la organización logren tomar conciencia de las responsabilidades que se van asumir si deciden que la empresa haga público una determinada normativa ética, es decir; el objetivo de la empresa al establecer un código ético es crear una cultura donde se delimiten las conductas deshonestas que podrían estar perjudicando al Talento Humano y a la organización, con la finalidad de marcar el camino a seguir por todos los miembros. Bajo esta perspectiva, la existencia de un código de ética en las empresas ayuda a mejorar la imagen y la credibilidad pública de las mismas, siendo esto un buen incentivo para su reputación.

No obstante, las empresas deben ser conscientes de los riesgos de prometer algo que no se pueda cumplir, pues en caso de no hacerlo, la desconfianza es mucho mayor, en virtud que "un código ético es fundamental en las relaciones en el ámbito empresarial; por ello, la normativa debe ser realista y coherente.

\section{Elementos constitutivos de la acción humana y su impacto en el individuo}


La afectividad humana, de acuerdo con lo planteado por Guillen (2006), es singular por estar unida a la capacidad de reflexión y a la voluntad libre; esta dimensión tiene relevancia en las decisiones humanas y constituye un eje fundamental para explicar su conducta.

Es así como según Seijo y Carvajal(2010), los sentimientos son importantes por la indudable influencia que tienen en el comportamiento y en cómo los mismos afectan las decisiones. Además de definirse como el estado de ánimo producido por causas que lo impresionan, tales como el entusiasmo ante un nuevo proyecto, el temor a una posible decisión en la organización, la alegría provocada por un acontecimiento positivo inesperado o el desánimo que genera la ausencia de resultados esperados.

Según Guillen (2006), los sentimientos pueden ir a favor o en contra de la voluntad y por tanto facilitar las acciones libres, pero el dominio de estos por parte de la voluntad y la razón, no está asegurado, los mismos serán buenos en la medida en que cooperen con las acciones buenas y a la inversa.

En este sentido, la acción humana, es en la medida en que supone una decisión libre de la voluntad, motivada por una intención que puede ser racional y emocional; es decir, aunque la acción es una, en ella se puede distinguir una intención que es el resultado de un juicio querido de la razón, acerca de la conveniencia de actuar o no, y afectado a la vez por los sentimientos. La intención marca la meta, el resultado o la consecuencia vinculada a dicha acción.

De acuerdo con lo mencionado, se ejemplifica al decir que se puede actuar con pocas fuerzas físicas (corporeidad) y además sin ilusión o sin ganas (afectividad); no obstante, esto no impide la responsabilidad ética si la acción fuera consciente (existe intención) y libre (decisión de la voluntad); de lo cual se deduce: no todo lo que se desea y ejecuta resulta conveniente.

También, junto a la intención y decisión de los medios para la puesta en práctica de la acción, existen unas circunstancias concretas, pues cada comportamiento, se produce en un momento, en un lugar y a partir de unos factores que afectan el mismo, y por tanto su juicio ético. En una organización éticamente enferma se pueden fomentar comportamientos deshonestos, mientras que en una saludable se puede contribuir con la mejora de sus miembros como personas, es decir, las circunstancias ambientales lógicamente afectan los comportamientos personales. 
Por último, toda acción humana tendrá como resultado unas consecuencias más o menos previsibles, por cuanto aunque ya no formen parte de la acción en sí, en la medida en que son resultado de la misma, pueden haber sido consideradas por quien actúa. En este enfoque, no debe olvidarse que toda acción humana tiene implicaciones sobre otras personas, pero principalmente en la que actúa, es decir, quien estafa se hace estafador y quien actúa sin hacer continua ostentación de sus éxitos se hace humilde.

Conviene destacar la necesidad de tener en cuenta los previsibles resultados de la actuación, por cuanto, toda acción acaba teniendo consecuencias sobre el actuante. Las personas que son afectadas por la decisión y de modo indirecto, por el efecto que el comportamiento provocado en terceros, tendrán en quien ha actuado, los resultados de sus propias acciones, denominadas también circunstancias que influyen en la valoración ética siempre y cuando sean previsibles y evitables.

Desde el punto de vista ético, la importancia de la intención para juzgar la acción en su conjunto, se entiende al analizar con detalle cuando se trata de medir las consecuencias del comportamiento humano. Si se miente, aunque no se note externamente, el ejecutor de la acción se hace mentiroso, si la intención es mala, quien la realiza está siendo malintencionado, aunque su actuación resulte un efecto externo positivo.

\section{Conclusiones}

Es importante que en el comportamiento de las personas en especial de los líderes que ocupan cargos de mayor responsabilidad en la organización se analice a partir de una perspectiva psicológica, por tal motivo; se identificó la relación del acoso laboral y el síndrome de cronos, ambos reconocidos como riesgos psicosociales que afectan la salud mental de los trabajadores, como la autoestima y la motivación, ello con la finalidad de no tener un buen desempeño y de esta forma incide negativamente en diversos objetivos organizacionales.

En este sentido, el miedo en el individuo producido a través del acoso laboral y el síndrome de cronos generan la pérdida del talento, en virtud que, causa enfermedades entre otras fuentes de estrés psicosocial. No obstante, sus consecuencias son dañinas para el trabajador, organización, y sociedad en general, por tal motivo, es cada vez más indispensable la necesidad con respecto a realizar intervenciones, que eviten este tipo de 
riesgos que afecta la reputación e integridad de la persona y de la organización.

De acuerdo a lo anterior, un ataque psicológico ocasionado por un jefe que implementa un liderazgo basado en el miedo causado por el síndrome de cronos; es así como el comportamiento del agresor obedece a una serie de disposiciones previas anormales o patológicas, con experiencias traumáticas causadas por el trastorno paranoide y antisocial en la personalidad que se reflejan a lo largo del tiempo no solamente en el ámbito laboral.

\section{Referencias bibliográficas}

Bedoya, C. \& Velásquez, A. (2014). Análisis de la incidencia del miedo en la organización desde la perspectiva psicobiológica. Revista Colombiana de Psicología, Vol. $23 N^{\circ}$ (2). https://dialnet.unirioja. es/descarga/articulo/5016329.pdf Universidad del Valle, Cali, Colombia.

Bustillo, F. (2011). El síndrome de Cronos. La nueva enfermedad Organizacional. Team Action. Entrenamiento y desarrollo. Extraído de http://www.teamaction.com. $\mathrm{ar} / \operatorname{archivos/cronos.pdf}$.

Brealey, S. \& Marcus. J. (2014). Principios de finanzas corporativas. 9na. ed. Editorial McGraw Hill.

Contreras, D. (2010). Síndrome de Cronos. Economía y Empresas. Diario On Line El Libre Pensador. 12/11/2010. Accedido el 25 de julio de 2016 Extraido de http:// www.ellibrepensador.com/2010/11/12/ sindrome-de-cronos/.

Cestau, D. (2002). Las diez causas principales del fracaso del liderazgo. De Gerencia. com. 21 de mayo de 2002. Accedido el 25 de julio de 2016. Extraído de: http://www. degerencia.com/articulo/las_diez_causas_ principales_del_fracaso_del_liderazgo.

Grettchen, S. (2011). Síndrome de Cronos. Revista Scielo. ISSN 0001-6002/. http:// www.scielo.sa.cr/pdf/amc/v53n3/art03. pdf. Vol. 5. $\mathrm{N}^{\circ} 3$.

Guillen, M. (2006). Ética y Práctica de la Responsabilidad Social Empresarial. Editorial Planeta Venezolana, S.A.

Hirtz, B. (2010). El miedo a ser reemplazados: El Síndrome de Cronos en la empresa. Accedido el 25 de julio de 2016 http:// www.buscarempleo.es/riesgos-laborales/ el-sindrome-de- cronos-en-la-empresa. html

Lozano, J. F. (2011). Qué es la ética de la empresa. Editorial Proteus. España.

Martínez, M. (2012). El acoso psicológico en el trabajo y mobbing: patología emergente. Gaceta Internacional de Ciencias Forenses. ISSN: 2174-9019. N³. http:// www.uv.es/gicf/3Ar1_Mtz_Leon_ GICF_03.pdf España: Universidad de Valladolid.

Martínez, M.; Muñiz, I.; Camino, L.; Torres, M. \& Burón, Q. (2012). El acoso psicológico en el trabajo o mobbing: patología emergente. Revista Ciencia forense ISSN: 2174-9019. http://www.uv.es/gicf/3Ar1_ Mtz_Leon_GICF_03.pdf

Sue, D. \& Sue, S. (2010). Psicopatología, Comprendiendo la Conducta Anormal. (9a ed.). Editorial México. Cengage Learning.

Seijo, C. \& Añez, N. (2011). La ética social y la dignificación de la vida humana: un enlace epistémico en la sociedad. Revista Clío América. Año 5 No. 9, p.p. $99-$ 111. http://www.centroetica.uct.cl/wp- 
content/uploads/2017/01/etica-social.pdf

Seijo, C. \& Carvajal, L. (2010). La ética como plataforma para la toma de decisiones en las empresas aseguradoras. Revista Innovación y Gerencia. Vol. I. No. 1. ISSN 1856-8807

Verona, M. \& Santana, M. (2012).

Consecuencias y Responsabilidades de la Empresa ante el Mobbing.

Revista Ciencia y Sociedad.Vol. 39. $N^{\circ} 3$. Instituto Tecnológico de Santo Domingo. República Dominicana. ISSN: 0378-7680. http://www.redalyc.org/ pdf/870/87031897002.pdf. 